\title{
Copy number gain of CMTM6 increases the expression of PD-L1 in undifferentiated pleomorphic sarcoma
}

\section{Shin Ishihara}

Graduate School of Medical Sciences, Kyushu University

Takeshi Iwasaki

Graduate School of Medical Sciences, Kyushu University

Kenichi Kohashi

Graduate School of Medical Sciences, Kyushu University

Yuichi Yamada

Graduate School of Medical Sciences, Kyushu University

Yu Toda

Graduate School of Medical Sciences, Kyushu University

Yoshihiro Ito

Graduate school of Medical Sciences, Kyushu University

Yousuke Susuki

Graduate School of Medical Sciences, Kyushu University

Kengo Kawaguchi

Graduate School of Medical Sciences, Kyushu University

Dai Takamatsu

Graduate School of Medical Sciences, Kyushu University

Shinichiro Kawatoko

Graduate School of Medical Sciences, Kyushu University

Daisuke Kiyozawa

Graduate School of Medical Sciences, Kyushu University

Taro Mori

Graduate School of Medical Sciences, Kyushu University

Izumi Kinoshita

Graduate School of Medical Sciences, Kyushu University

Hidetaka Yamamoto

Graduate School of Medical Sciences, Kyushu University

Toshifumi Fujiwara

Graduate School of Medical Sciences, Kyushu University

Nokitaka Setsu 
Graduate School of Medical Sciences, Kyushu University

\section{Makoto Endo}

Graduate School of Medical Sciences, Kyushu University

\section{Yoshihiro Matsumoto}

Graduate School of Medical Sciences, Kyushu University

\section{Yasuharu Nakashima}

Graduate School of Medical Sciences, Kyushu University

Yoshinao Oda ( $D$ oda@surgpath.med.kyushu-u.ac.jp)

Graduate School of Medical Sciences, Kyushu University https://orcid.org/0000-0001-9636-1182

\section{Original Article - Cancer Research}

Keywords: PD-L1, CMTM6, copy number, prognosis, Undifferentiated pleomorphic sarcoma

Posted Date: February 10th, 2021

DOl: https://doi.org/10.21203/rs.3.rs-201625/v1

License: (c) (i) This work is licensed under a Creative Commons Attribution 4.0 International License. Read Full License

Version of Record: A version of this preprint was published at Journal of Cancer Research and Clinical Oncology on April 3rd, 2021. See the published version at https://doi.org/10.1007/s00432-021-03616-4. 


\section{Abstract \\ Background}

Undifferentiated pleomorphic sarcoma (UPS) is a sarcoma with a poor prognosis. A clinical trial, SARC028, revealed that treatment with anti-PD-1 drugs was effective against UPS. Studies have reported that UPS expresses PD-L1, sometime strongly ( $\geq 50 \%$ ). However, the mechanism of PD-L1 expression in UPS has remained still unclear. CKLF-like MARVEL transmembrane domain containing 6 (CMTM6) was identified as a novel regulator of PD-L1 expression. The positive relationship between PD-L1 and CMTM6 has been reported in several studies. The aim of this study was to examine CMTM6 expression in UPS and evaluate the relationship between PD-L1 and CMTM6.

\section{Materials and methods}

Fifty-one primary UPS samples were subjected to CMTM6 and PD-L1 immunostaining. CMTM6 expression was assessed using proportion and intensity scores. CMTM6 gene copy number was also evaluated using a real-time PCR-based copy number assay. We also analyzed the mRNA expression and copy number variation of PD-L1 and CMTM6 in The Cancer Genome Atlas (TCGA) data.

\section{Results}

TCGA data indicated that the mRNAs encoded by genes located around 3p22 were coexpressed with CMTM6 mRNA in UPS. Both proportion and intensity scores of CMTM6 positively correlated with strong PD-L1 expression ( $\geq 50 \%$ ) (both $p=0.023$ ). CMTM6 copy number gain increased CMTM6 expression. Patients with UPS with a high CMTM6 intensity score had worse prognosis for overall survival.

\section{Conclusions}

CMTM6 expression was significantly correlated with PD-L1 expression. CMTM6 expression induced strong PD-L1 expression ( $\geq 50 \%$ ). CMTM6 copy number gain promoted CMTM6 expression and increased PD-L1 expression in UPS.

\section{Introduction}

Undifferentiated pleomorphic sarcoma (UPS) is a sarcoma with a poor prognosis, the tumors of which do not show specific differentiation. It is diagnosed by excluding other sarcomas. It is usually treated with excision, chemotherapy, and radiation therapy, but its prognosis is still unfavorable (WHO classification of Tumours of Soft Tissue and Bone, 2020). A clinical trial (SARC028) reported that treatment with antiPD-1 therapy was beneficial for the survival of UPS patients (Tawbi et al. 2017). In addition, some investigations demonstrated that UPS cells express PD-L1 (Boxberg et al. 2018; Keung et al. 2020; 
Ishihara et al. 2020). In detail, 30\% or more of UPS cases were found to express PD-L1 (tumor proportion cut-off $\geq 1 \%$ ), while approximately $10 \%$ of them strongly express PD-L1 (tumor proportion cut-off $\geq 50 \%$ ) (Boxberg et al. 2018; Ishihara et al. 2020). PD-L1 expression is regulated by IFN-y secreted by immune cells (Schalper et al. 2017; Burr et al. 2017a; Mezzadra et al. 2017; Ishihara et al. 2020). We reported that IFN-y induces PD-L1 expression in UPS and that PD-L1 expression (cut-off $\geq 1 \%$ ) in UPS positively correlated with the level of tumor-infiltrating lymphocytes (TILs) (Ishihara et al. 2020). However, in our previous study, cases with PD-L1 expression ( $\geq 50 \%$ ) were not significantly associated with TILs (Boxberg et al. 2018), suggesting that factors other than TILs also regulate PD-L1 expression. Boxberg et al. investigated the association between increased copy number of PD-L1 and PD-L1 expression but did not find a significant correlation (Ishihara et al. 2020).

It has been reported that CKLF-like MARVEL transmembrane domain containing 6 (CMTM6) protects PDL1 from lysosomal degradation and promotes its expression on the cell membrane (Burr et al. 2017b; Mezzadra et al. 2017). CMTM6 acts on PD-L1 by a different route from the stimulation by IFN-y (Burr et al. 2017b; Mezzadra et al. 2017). A study on non-small-cell lung cancer reported that CMTM6 expression positively correlated with PD-L1 expression and that CMTM6 was a predictor of the therapeutic effect of an anti-PD-1 drug (Koh et al. 2019). In addition, in cancers such as hepatocellular carcinoma and gastric cancer, CMTM6 has been reported to be coexpressed with PD-L1 (Li et al. 2020; Liu et al. 2020).

The purpose of this study is to evaluate the expression of CMTM6 in UPS and to assess the relationship between PD-L1 and CMTM6. In addition, The Cancer Genome Atlas (TCGA) data on CMTM6 were analyzed and the correlation of CMTM6 and PD-L1 was assessed.

\section{Materials And Methods}

\section{Patients and materials}

This study was conducted in accordance with the principles of the Declaration of Helsinki. It was also approved by the Ethics Committee of Kyushu University (Nos. 29-429, 29-625) and consent was obtained from patients who donated tissue. A total of 51 UPS cases, diagnosed as malignant fibrous histiocytoma or UPS, were retrieved from among soft-tissue tumors registered at the Department of Anatomic Pathology, Graduate School of Medical Sciences, Kyushu University, Fukuoka, Japan, from 1998 to 2019.

To collect definitive UPS samples, secondary sarcomas, radiation-associated sarcomas, myxofibrosarcomas, dedifferentiated liposarcomas, and other sarcomas were excluded (Ishihara et al. 2020). A total of 48 tumors were the same as in this previous study, while three were newly retrieved. Four tumors that had been used in the previous study were not used in this study because the formalin-fixed, paraffin-embedded (FFPE) samples had been used up (Ishihara et al. 2020).

Immunohistochemistry 
FFPE tissue was sliced into sections at a thickness of $3 \mu \mathrm{m}$. Antigen retrieval was performed by boiling the slides in $10 \mathrm{mM}$ sodium citrate ( $\mathrm{pH} \mathrm{6.0)}$ ) or Target Retrieval Solution (Dako). The immunoperoxidase polymer method (EnVision-kit and EnVision Flex-kit; Dako) was used. We used the following primary antibodies: anti-PD-L1 (rabbit, polyclonal, 28-8, 1:400; Abcam) and anti-CMTM6 (rabbit, polyclonal, SAB270119, 1:100; Sigma-Aldrich). We stained PD-L1 and CMTM6 using the EnVision Flex-kit and EnVision-kit, respectively.

PD-L1 was assessed by determining the proportion of membranous staining-positive tumor cells relative to all tumor cells, as described previously (Ishihara et al. 2020). CMTM6 evaluation was performed in a manner similar to that used for estrogen and progesterone receptor evaluation in breast cancer using the Allred score. Proportion score (PS) was assessed by determining the proportion of tumor cells revealing cytoplasmic positivity for staining relative to all tumor cells on the slide. PS of CMTM6 was defined as follows: PS 0: no positive cells, PS 1: $>0 \%$ and $<1 \%$ positive tumor cells, PS $2: \geq 1 \%$ and $<10 \%$, PS $3: \geq$ $10 \%$ and $<33 \%$, PS $4: \geq 33 \%$ and $<66 \%$, and PS $5: \geq 66 \%$ and $\leq 100 \%$. Intensity score (IS) was assessed using the intensity of the staining as follows: IS 0 : negative, not stained, IS 1: weak cytoplasmic staining and no membranous staining, and IS 2: strong cytoplasmic and strong membranous staining. In the Allred scoring system, IS is classified into four groups: "negative," "weak," "intermediate," and "strong." However, in this study, cases were classified into three groups to maintain reproducibility, because it was difficult to distinguish between "intermediate" and "strong". We set the cut-offs of PS and IS of CMTM6 immunostaining for statistical analysis by drawing ROC curves.

\section{Copy number assay}

Copy number assay was conducted using TaqMan quantitative PCR (Thermo Fisher Scientific Inc.). We used the following primers: CMTM6 (catalog number: 4400291; Thermo Fisher Scientific Inc.) and RNase $P$ (catalog number: 4403326; Thermo Fisher Scientific Inc.). For the copy number assay, 13 frozen samples and 14 FFPE samples were used. The copy number assay was not conducted for some cases because the sample volume was small or little sample was left. A normal tissue sample of a UPS case was also tested as a control. Finally, DNA was extracted in 28 cases. PCR was performed with THUNDERBIRD Probe qPCR Mix (TOYOBO), using the following protocol on a Step One Plus Real Time PCR System (Thermo Fisher Scientific Inc.): pre-denaturing at $95^{\circ} \mathrm{C}$ for $10 \mathrm{~min}$; and then 45 cycles of denaturing at $98^{\circ} \mathrm{C}$ for $15 \mathrm{~s}$, annealing at $60^{\circ} \mathrm{C}$ for $15 \mathrm{~s}$, and extension at $68^{\circ} \mathrm{C}$ for $30 \mathrm{~s}$. The obtained relative quantities were processed using CopyCaller (Thermo Fisher Scientific Inc.) and copy numbers were determined.

Subgrouping in accordance with PD-L1, CMTM6, and tumor-infiltrating lymphocytes

We divided the UPS cases into subgroups according to the PD-L1 expression, the level of CD8-positive TILs, and the CMTM6 expression. In this subgrouping, the cut-off of PD-L1 was set as $1 \%$ because this is the threshold for administering anti-PD-1 therapy for many cancers. We counted CD8-positive TILs in UPS in five randomly chosen high-power fields, as previously described (Ishihara et al. 2020). Cases were classified as TILs-high or TILs-low according to the cut-off, which was determined by drawing ROC curves 
(Supplementary Figure 1). If either PS or IS of CMTM6 was evaluated as high, CMTM6 expression was considered to be positive.

\section{DNA copy number and mRNA expression profiling using TCGA data}

DNA copy number data and mRNA expression data of 50 UPS cases in TCGA

(https://www.cancer.gov/tcga) were analyzed using the cBio Cancer Genomics Portal (cBioPortal), an open platform for evaluating genomic data (http://www.cbioportal.org/) (Gao et al. 2014; Cerami et al. 2017).

\section{Statistics}

The relationship between the immune expression of CMTM6 and PD-L1 was analyzed by Fisher's exact test. Steel's multiple comparison test was used to analyze the correlation between CMTM6 mRNA and DNA copy number in TCGA data using cBioPortal. The relationship between immunohistochemistry (IHC) data and DNA copy number of CMTM6 was analyzed using the Mann-Whitney U test. Survival curves were constructed using the Kaplan-Meier method. Overall survival and disease-free survival curves were analyzed using the log-rank test. Multivariate analysis was conducted using Cox's proportional hazard model. We used the JMP statistical software package (version 14; SAS Institute) for analysis. A $p$-value of $<0.05$ was considered significant in the statistical analysis.

\section{Results}

\section{TCGA data analysis}

The analyzed data are presented in Figure 1. There was a positive correlation between the mRNA expression of $P D-L 1$ and that of $C M T M 6\left(p=0.0431\right.$ and $R^{2}=0.08$, Figure $\left.1 \mathrm{a}\right)$. A list of the genes encoding mRNAs coexpressed with CMTM6 is shown in Figure $1 \mathrm{~b}$. The genes most closely related to CMTM6 are presented at the top. Many genes located around 3p22 on the short arm of chromosome 3 are listed. The copy number segment of UPS cases is shown in Supplementary Figure 2. The relationship between mRNA expression and copy number variation of CMTM6 is presented in Figure 1c. The mRNA expression of CMTM6 in UPS with CMTM6 copy number gain increased compared with that in UPS with diploid CMTM6 copy number (shallow deletion vs. diploid: $p=0.112$, diploid vs. gain: ${ }^{*} p=0.025$ ).

\section{Clinicopathological data analysis}

The clinicopathological data of the retrieved UPS cases are summarized in Table 1. A total of 27 (52.9\%) cases were younger than 69 years of age and $24(47.1 \%)$ cases were 69 years of age or older. A total of $27(52.9 \%)$ cases were male and $24(47.1 \%)$ were female. Data on tumor size were available for 43 cases: $12(27.9 \%)$ cases had tumors of $5 \mathrm{~cm}$ or smaller and $31(72.1 \%)$ had tumors larger than $5 \mathrm{~cm}$. Three $(5.9 \%)$ cases arose in the soft tissues of the head and neck, nine (17.6\%) in the upper extremity, eight 
(15.7\%) in the trunk, and $31(60.8 \%)$ in the lower extremity. A total of $26(51 \%)$ and $25(49 \%)$ cases were evaluated as FNCLCC grades 2 and 3, respectively.

\section{Immunohistochemical analysis}

The results of IHC for CMTM6 and PD-L1 are summarized in Table 2. The representative feature of UPS, namely, pleomorphism, haphazard arrangement, and bizarre giant cells, are shown in Figure $2 \mathrm{a}$ and $\mathrm{b}$. Photographs of IHC for CMTM6 and PD-L1 are shown in Figure $2 \mathrm{c}-\mathrm{e}$ and $2 \mathrm{f}-\mathrm{h}$, respectively. For the PS of CMTM6, 2/51 (3.9\%) cases were classified as PS0, 3/51 (5.9\%) as PS1, 22/51 (43.1\%) as PS2, 18/51 (35.3\%) as PS3, 6/51 (11.8\%) as PS4, and none as PS5. Regarding the IS of CMTM6, 2/51 (3.9\%) cases were classified as IS0, 24/51 (47.1\%) as IS1, and 25/51 (49\%) as IS2. Regarding PD-L1, 31/51 (60.8\%) cases did not express PD-L1, 15/51 (29.4\%) expressed focal PD-L1 ( $\geq 1$ and $<50 \%$ ), and 5/51 (9.8\%) strongly expressed PD-L1 ( $\geq 50 \%)$.

\section{The relationship between CMTM6 and PD-L1 expression in IHC}

The relationship between CMTM6 and PD-L1 expression is shown in Supplementary Figure 3. The cutoffs of PS and IS of CMTM6 were determined by drawing ROC curves (Supplementary Figure 4). On the AUC of the curves, it was when CMTM6 surrogated strong PD-L1 $(\geq 50 \%)$ rather than PD-L1 $(>1 \%)$ that PS and IS of CMTM6 became a highly specific factor. Therefore, cut-offs for determining strong PD-L1 $(\geq$ $50 \%$ ) were used; the cut-offs for PS and IS were 3 and 2, respectively. The relationships between PS and IS of CMTM6 and PD-L1 are summarized in Table 3. Positive correlations between the expression of PDL1 $(\geq 50 \%)$ and PS or IS of CMTM6 were seen (both * $p=0.023$ ). The correlations between the expression of PD-L1 ( $\geq 1 \%)$ and PS or IS of CMTM6 were not significant.

\section{CMTM6 copy number analysis}

CMTM6 copy number in UPS is presented in Figure 3a. Results were obtained for $14 / 28$ cases, with 14 cases not being properly tested due to sample defects. Normal tissue was tested as a control. CMTM6 copy numbers ranged from 1 to 13 (mean 3.6). IHC data for PD-L1 and CMTM6 are shown below. The relationship between CMTM6 copy number, obtained through qPCR, and CMTM6 protein expression, revealed by IHC, was analyzed (Figure $3 b$ and $3 c$ ). CMTM6 copy number significantly correlated with the PS of CMTM6 $\left({ }^{\star} p=0.030\right)$. CMTM6 copy number was not significantly associated with the IS of CMTM6.

\section{Survival analysis}

The outcome of survival analysis is shown in Figure 4. Prognostic information for 41 cases was available. Although patients with UPS and higher PS of CMTM6 did not have worse prognosis for overall survival (Figure 4a, $p=0.169$ ), UPS patients with higher IS of CMTM6 did have worse prognosis (Figure $4 \mathrm{~b}, p=0.048)$. PS and IS of CMTM6 did not have significant associations with disease-free survival (Figure $4 \mathrm{c}, p=0.240$; and Figure $4 \mathrm{~d}, p=0.197$ ). Multivariate analysis and hazard ratio of overall survival are shown in Supplementary Table 1. CMTM6 IS was a significant factor for worse prognosis of overall survival in patients with UPS (hazard ratio: $20.3,95 \% \mathrm{Cl}: 2.10-197, p=0.009$ ). 
Figure 5 shows the subgrouping of UPS according to the level of CD8-positive TILs and CMTM6 expression. Among the 20 cases expressing PD-L1 ( $\geq 1 \%$ ), 3 cases expressed CMTM6 but had low CD8positive TILs, 11 cases expressed CMTM6 and had high CD8-positive TILs, and 6 cases did not express CMTM6 but had high CD8-positive TILs.

\section{Discussion}

UPS cells express PD-L1 and the PD-L1 expression is regulated by IFN- $\gamma$ secreted by immune cells (Schalper et al. 2017; Burr et al. 2017a; Mezzadra et al. 2017; Ishihara et al. 2020). IFN-y induces PD-L1 expression and that PD-L1 expression (cut-off $\geq 1 \%$ ) positively correlate with the level of TILs. The same phenomenon was noted in UPS. However, our previous study suggested that factors other than TILs also regulate PD-L1 expression. In the current study, we revealed the positive correlation between PD-L1 expression and CMTM6 expression.

CMTM6 expression was here shown to be positively correlated with PD-L1 expression in UPS. This finding is similar to those in previous reports on lung cancer, hepatocellular carcinoma, and gastric cancer (Koh et al. 2019; Li et al. 2020; Liu et al. 2020). CMTM6 has been reported to inhibit its lysosomal degradation, possibly by preventing its ubiquitination, and to promote PD-L1 expression on the cell membrane (Burr et al. 2017a; Mezzadra et al. 2017). In the current study, the same mechanism was also considered to be involved, in that CMTM6 prevented the lysosomal degradation of PD-L1 and assisted its membranous expression. Interestingly, CMTM6 expression closely and positively correlated with the strong expression of PD-L1 ( $\geq 50 \%$ ). All five UPS cases with strong PD-L1 expression ( $\geq 50 \%$ ) exhibited high CMTM6 expression. In UPS, like in other cancers, PD-L1 is induced by IFN-Y (Burr et al. 2017b; Schalper et al. 2017; Mezzadra et al. 2017; Ishihara et al. 2020). However, our findings suggest that strong PD-L1 expression in UPS requires high CMTM6 expression, in addition to the stimulation of IFN- $\gamma$.

CMTM6 copy number was found to be positively correlated with CMTM6 expression in the current study. Analysis of TCGA data revealed that the mRNAs encoded by some genes located around 3p22 were coexpressed with CMTM6 mRNA. UPS is known to have a complex karyotype, with chromosome numbers ranging from haploid to polyploid (WHO classification of Tumours of Soft Tissue and Bone, 2020). Data on mRNA expression suggested that the copy number of the genes located around 3p22 including CMTM6 often varies because of the chromosomal instability of UPS and that the impact of copy number variation of CMTM6 on its mRNA expression would be greater in UPS than that of other factors. It is possible that UPS cases have CMTM6 copy number variation, sometimes exhibit copy number gain, have upregulated CMTM6 expression, and strongly express PD-L1.

Tumors with positivity for CMTM6 and a low level of CD8-positive TILs were identified in the present study. It is generally considered necessary for tumor immunity that tumor cells express neoantigen(Li et al. 2020)(Gao et al. 2014)(Cerami et al. 2017). However, it is unclear whether or not UPS cases with PD-L1 expression, high CMTM6 expression, and low CD8-positive TILs express neoantigen; therefore, the effect 
of anti-PD-1 drugs on UPS should be evaluated from the perspective of CMTM6 expression. In addition, when using anti-PD-1 drugs for such tumors, it may be desirable to use drugs that promote cancer immunity, such as anti-CTLA4, as activators of the immune system. CMTM6 protein is suggested as a useful prognostic marker for the effect of anti-PD-1 drug in patients with non-small-cell lung cancer (Schalper et al. 2017). The same would also be true for UPS, a tumor with copy number variation of CMTM6. The current study could provide a basis for predicting the efficacy of anti-PD-1 therapy in UPS by evaluating CMTM6 expression.

Analysis of TCGA data revealed that the levels of PD-L1 and CMTM6 mRNAs were significantly positively correlated in UPS, which is consistent with the findings in lung cancer (Schalper et al. 2017). However, this correlation cannot be explained by the mechanism that CMTM6 assists PD-L1 to avoid lysosomal degradation. Instead, it is possible that there is some transcriptional regulator in common between PD-L1 and CMTM6, but this remains unknown. Strong immunostaining of CMTM6 was a poor prognostic factor in the current study. Therefore, CMTM6 inhibition may improve the prognosis of patients with UPS. However, CKLF-like MARVEL transmembrane domain containing 4 (CMTM4) was reported to interact with PD-L1 and restore PD-L1 expression in CMTM6-deficient melanoma cells (Mezzadra et al. 2017). Thereby, when using an anti-CMTM6 drug, the use of an anti-CMTM4 drug in combination is suggested.

In conclusion, in this study CMTM6 expression was found to be significantly correlated with PD-L1 expression. CMTM6 expression induced strong PD-L1 expression ( $\geq 50 \%$ ). CMTM6 copy number gain promoted CMTM6 expression and increased PD-L1 expression in UPS.

\section{Abbreviations}

CMTM6, CKLF like MARVEL transmembrane domain containing 6; FFPE, Formalin-fixed, paraffinembedded; IS, Intensity score; PD-L1, Programmed death-ligand 1; PS, Proportion score; TCGA, The Cancer Genome Atlas; TIL, Tumor-infiltrating lymphocytes; UPS, Undifferentiated pleomorphic sarcoma

\section{Declarations}

\section{Acknowledgment}

The results shown here are in part based upon data generated by the TCGA Research Network: https://www.cancer.gov/tcga a

We appreciate M. Tomita and M. Nakamizo at the Department of Anatomic Pathology, Kyushu University for providing technical support. We additionally thank the Research Support Center, Graduate School of Medical Sciences, Kyushu University, for providing the experimental devices.

\section{Funding}


The present study was supported by the Japan Society for the Promotion of Science KAKENHI (19H03444).

\section{Conflicts of interest}

The authors declare that they have no competing interests

\section{Ethics approval}

The present study was approved by the Kyushu University Committee of Bioethics (approval no. 29-429 and 29-625; 2017).

\section{Consent to participate}

Informed consent was obtained from all participants included in the study.

\section{Authors' contributions}

Authors' contributions $\mathrm{SI}, \mathrm{TI}, \mathrm{KK}, \mathrm{HY}$ and $\mathrm{YO}$ designed this study and wrote the manuscript. SI, $\mathrm{TI}$ performed the experiments. SI, YT, YI, YS, TM, SK, DT, IK, TM, DK, TF, NS, ME, YM and YN collected the materials. SI, TI, KK, YY IK and YO performed histological re-evaluation of the samples and confirmed the diagnosis. $\mathrm{SI}, \mathrm{TI}$ and $\mathrm{YO}$ supervised the experiments.

\section{References}

Boxberg M, Steiger K, Lenze U, et al (2018) PD-L1 and PD-1 and characterization of tumor-infiltrating lymphocytes in high grade sarcomas of soft tissue-prognostic implications and rationale for immunotherapy. Oncoimmunology 7:1-11. https://doi.org/10.1080/2162402X.2017.1389366

Burr ML, Sparbier CE, Chan Y-C, et al (2017a) CMTM6 maintains the expression of PD-L1 and regulates anti-tumour immunity. Nature 549:101-105. https://doi.org/10.1038/nature23643

Burr ML, Sparbier CE, Chan YC, et al (2017b) CMTM6 maintains the expression of PD-L1 and regulates anti-Tumour immunity. Nature 549:101-105. https://doi.org/10.1038/nature23643

Cerami et al. (2017) The CBio Cancer Genomics. Cancer Discov 32:736-740. https://doi.org/10.1158/2159-8290.CD-12-0095.The

Gao J, Aksoy BA, Dogrusoz U, et al (2014) Integrative Analysis of Complex Cancer Genomics and Clinical Profiles Using the cBioPortal Complementary Data Sources and Analysis Options. Sci Signal 6:1-20. https://doi.org/10.1126/scisignal.2004088.Integrative

Ishihara S, Yamada Y, Iwasaki T, et al (2020) PD-L1 and IDO-1 expression in undifferentiated pleomorphic sarcoma: The associations with tumor infiltrating lymphocytes, dMMR and HLA class I. Oncol Rep. https://doi.org/10.3892/or.2020.7837 
Keung EZ, Burgess M, Salazar R, et al (2020) Correlative analyses of the SARC028 trial reveal an association between sarcoma-associated immune infiltrate and response to pembrolizumab. Clin Cancer Res 26:1258-1266. https://doi.org/10.1158/1078-0432.CCR-19-1824

Koh YW, Han JH, Haam S, et al (2019) Increased CMTM6 can predict the clinical response to PD-1 inhibitors in non-small cell lung cancer patients. Oncoimmunology 8:1-7.

https://doi.org/10.1080/2162402X.2019.1629261

Li X, Chen L, Gu C, et al (2020) CMTM6 significantly relates to PD-L1 and predicts the prognosis of gastric cancer patients. PeerJ 8:1-13. https://doi.org/10.7717/peerj.9536

Liu LL, Zhang SW, Chao X, et al (2020) Coexpression of CMTM6 and PD-L1 as a predictor of poor prognosis in macrotrabecular-massive hepatocellular carcinoma. Cancer Immunol Immunother. https://doi.org/10.1007/s00262-020-02691-9

Mezzadra R, Sun C, Jae LT, et al (2017) Identification of CMTM6 and CMTM4 as PD-L1 protein regulators. Nature 549:106-110. https://doi.org/10.1038/nature23669

Schalper KA, Carvajal-Hausdorf D, McLaughlin J, et al (2017) Differential expression and significance of PD-L1, IDO-1, and B7-H4 in human lung cancer. Clin Cancer Res 23:370-378. https://doi.org/10.1158/1078-0432.CCR-16-0150

Tawbi HA, Burgess M, Bolejack V, et al (2017) Pembrolizumab in advanced soft-tissue sarcoma and bone sarcoma (SARC028): a multicentre, two-cohort, single-arm, open-label, phase 2 trial. Lancet Oncol 18:1493-1501. https://doi.org/10.1016/S1470-2045(17)30624-1

\section{Tables}




\begin{tabular}{|c|c|c|}
\hline \multicolumn{3}{|c|}{ Table 1. Clinicopathologic data } \\
\hline \multicolumn{3}{|l|}{ Feature } \\
\hline \multirow[t]{2}{*}{ Age } & $<69$ & $27 / 51(52.9 \%)$ \\
\hline & $\geq 69$ & $24 / 51(47.1 \%)$ \\
\hline \multirow[t]{2}{*}{ Sex } & M & $27 / 51(52.9 \%)$ \\
\hline & $\mathrm{F}$ & $24 / 51(47.1 \%)$ \\
\hline \multirow[t]{2}{*}{ Size } & $\leq 5 \mathrm{~cm}$ & $12 / 43(27.9 \%)$ \\
\hline & $>5 \mathrm{~cm}$ & $31 / 43(72.1 \%)$ \\
\hline \multirow[t]{4}{*}{ Location } & Head and Neck & $3 / 51(5.9 \%)$ \\
\hline & Upper Extremity & $9 / 51(17.6 \%)$ \\
\hline & Trunk & $8 / 51(15.7 \%)$ \\
\hline & Lower Extremity & $31 / 51(60.8 \%)$ \\
\hline \multirow[t]{2}{*}{ FNCLCC } & Grade 2 & $26(51.0 \%)$ \\
\hline & Grade 3 & $25(49.0 \%)$ \\
\hline
\end{tabular}




\begin{tabular}{|c|c|c|}
\hline \multicolumn{2}{|c|}{ Table 2: The ratio of positive immunostain } & $\mathrm{n} / 51(\%)$ \\
\hline CMTM6 PS & $0: 0 \%$ & $2(3.9 \%)$ \\
\hline & $1: 0 \leq$ and $<1 \%$ & $3(5.9 \%)$ \\
\hline & $2: 1 \leq$ and $<10 \%$ & $22(43.1 \%)$ \\
\hline & $3: 10 \leq$ and $<33 \%$ & $18(35.3 \%)$ \\
\hline & 4: $33 \leq$ and $<66 \%$ & $6(11.8 \%)$ \\
\hline & 5: $66 \% \leq$ and $\leq 100 \%$ & $0(0 \%)$ \\
\hline CMTM6 IS & 0 : negative & $2(3.9 \%)$ \\
\hline & 1: weak & $24(47.1 \%)$ \\
\hline & 2: strong & $25(49.1 \%)$ \\
\hline PD-L1 & $<1 \%$ & $31(60.8 \%)$ \\
\hline & $1 \leq$ and $<50 \%$ & $15(29.4 \%)$ \\
\hline & $\geq 50 \%$ & $5(9.8 \%)$ \\
\hline
\end{tabular}

Table 3. The correlation of PD-L1 and CMTM6

CMTM6

PS IS

$\begin{array}{llll}0,1,2 & 3,4,5 & 0,1 & 2\end{array}$

$\begin{array}{lllll}<1 \% & 18(35.3 \%) & 13(25.5 \%) & 17(33.3 \%) & 14(27.5 \%) \\ 1 \% \leq,<50 \% & 8(15.7 \%) & 7(13.7 \%) & 9(17.6 \%) & 6(11.8 \%) \\ \geq 50 \% & 0 & 5(9.8 \%) & 0 & 5(9.8 \%)\end{array}$

PD-L1

$50 \%$ cut off $\quad p=0.023^{*} \quad p=0.023^{*}$

$1 \%$ cut off 


\section{Supplemental Data}

Supplemental figures and tables are not available with this version

Figures

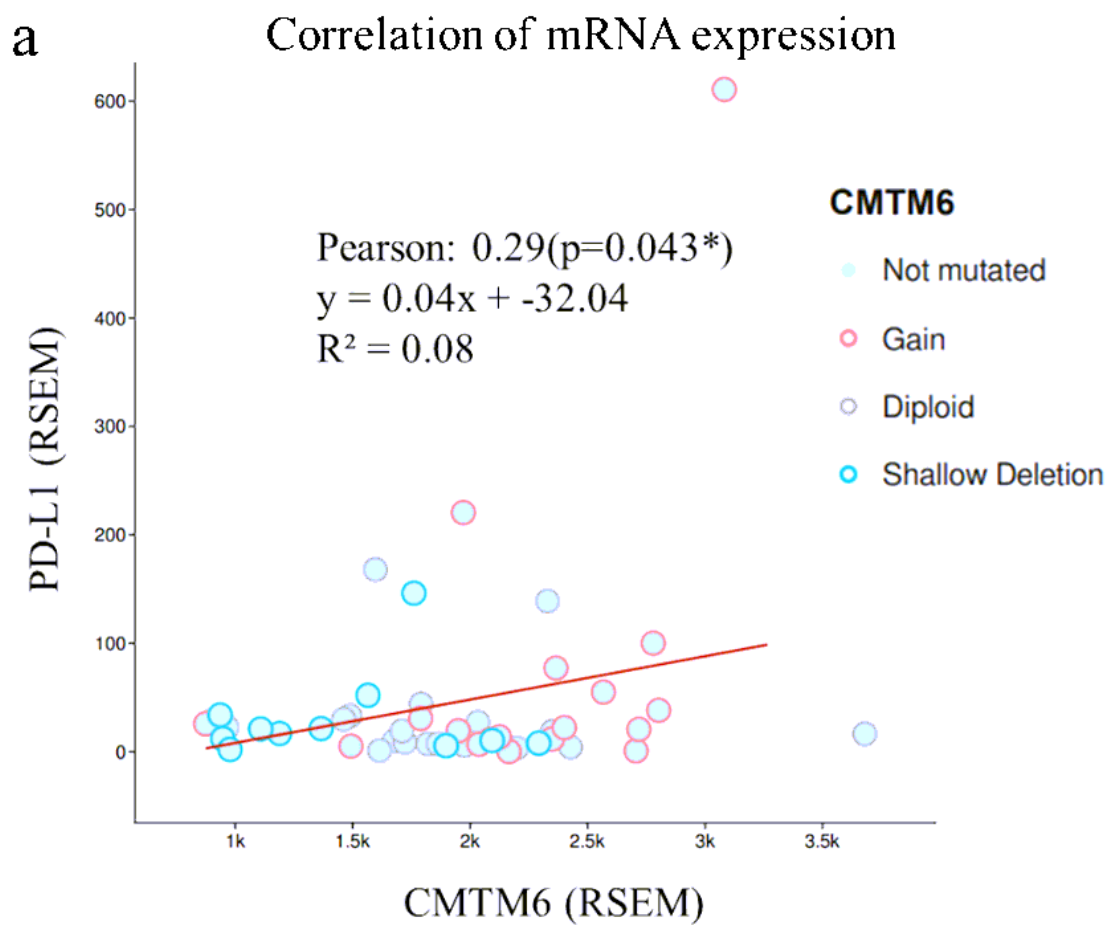

b Genes of which mRNA coexpressed with CMTM6

\begin{tabular}{|c|c|c|c|c|}
\hline Correlated Gene & Locus & Corelation & pvalue & qvalue \\
\hline RAB5A & $3 p 24.3$ & 0.660 & $1.85 \mathrm{e}-7$ & $2.259 e-3$ \\
\hline PDCDGIP & $3 p 22.3$ & 0.656 & $2.27 \mathrm{e}-7$ & $2.259 \mathrm{e}-3$ \\
\hline BHLHE40 & $3 p 26.1$ & 0.643 & $4.89 \mathrm{e}-7$ & $3.238 \mathrm{e}-3$ \\
\hline MLH1 & $3 \mathrm{p} 22.2$ & 0.608 & $2.817 \mathrm{e}-6$ & 0.0140 \\
\hline EAF1 & $3 p 25.1$ & 0.601 & $3.993 e-6$ & 0.0146 \\
\hline GLB1 & $3 p 22.3$ & 0.599 & $4.405 \mathrm{e}-6$ & 0.0146 \\
\hline MFSD14B & $9 q 22.32$ & 0.593 & $5.712 e-6$ & 0.0162 \\
\hline BAALC-AS2 & $8 q 22.3$ & -0.571 & $1.472 \mathrm{e}-5$ & 0.0366 \\
\hline FKBP1AP1 & $19 q 13.43$ & 0.567 & $1.751 \mathrm{e}-5$ & 0.0386 \\
\hline NGLY1 & $3 p 24.2$ & 0.547 & $3.968 \mathrm{e}-5$ & 0.0761 \\
\hline UBP1 & $3 p 22.3$ & 0.545 & $4.213 e-5$ & 0.0761 \\
\hline TMEM186 & $16 \mathrm{p} 13.2$ & -0.537 & $5.722 \mathrm{e}-5$ & 0.0879 \\
\hline
\end{tabular}

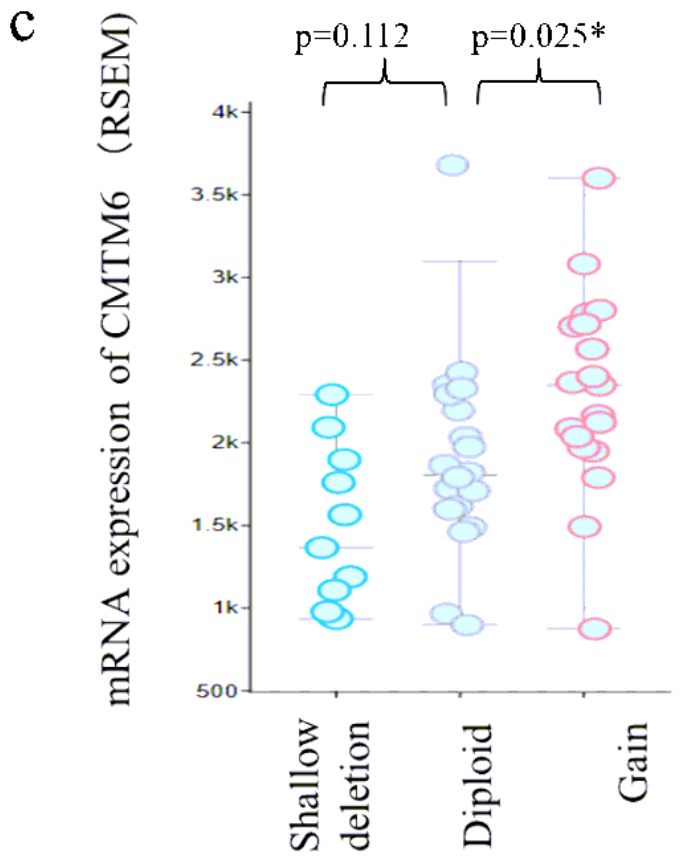

CNV of CLTM6 in UPS

Figure 1 
Data obtained from The Cancer Genome Atlas (TCGA). (a) The mRNAs of PD-L1 and CKLF-like MARVEL transmembrane domain containing 6 (CMTM6) (RSEM) correlate positively. (b) Genes coexpressed with CMTM6. (c) The relationship between the mRNA expression and copy number variation of CMTM6.

a

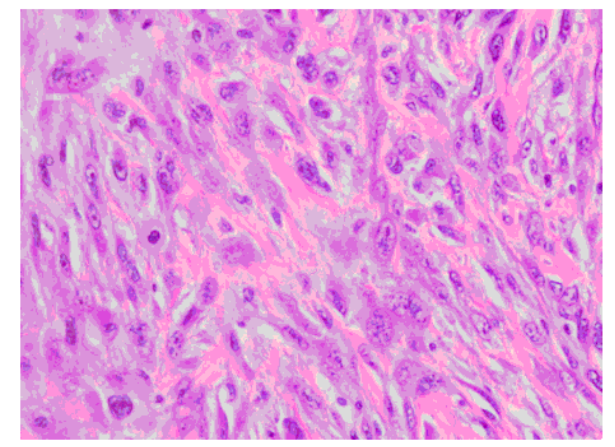

Pleomorphic and haphazard

$\mathrm{c}$

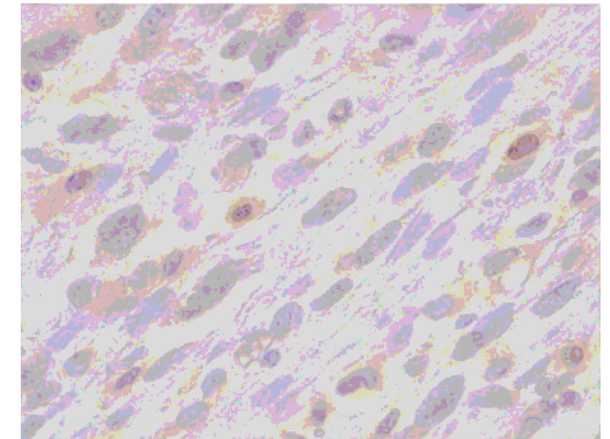

IS: 0

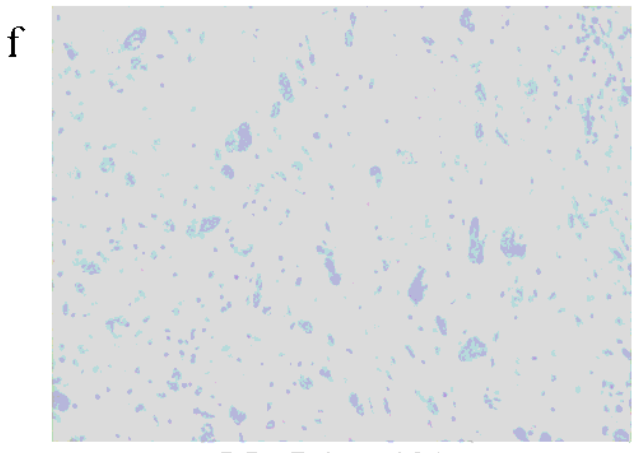

PD-L1: $<1 \%$

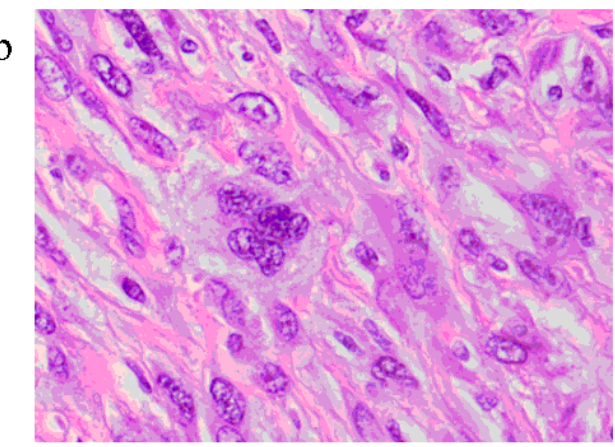

Bizarre giant cell

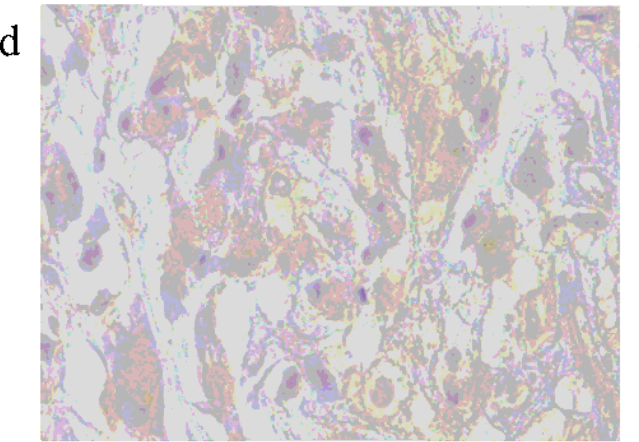

IS: 1

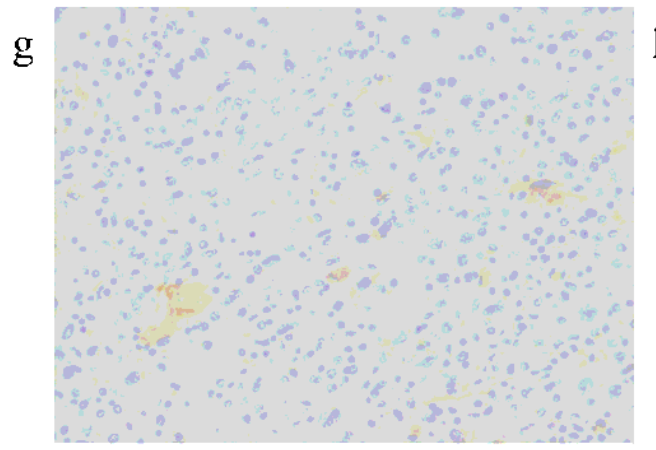

PD-L1: $1 \% \leq$ and $<50 \%$

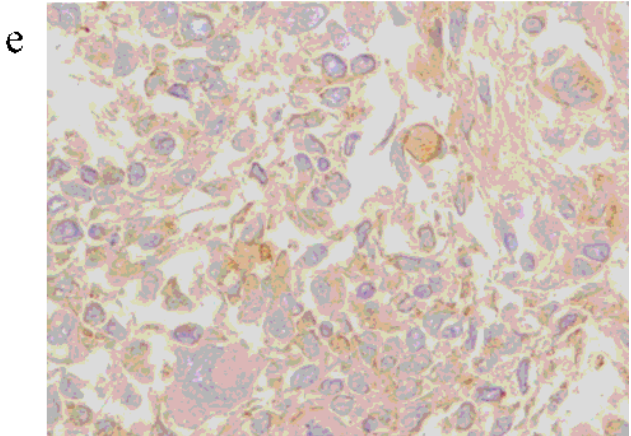

IS: 2

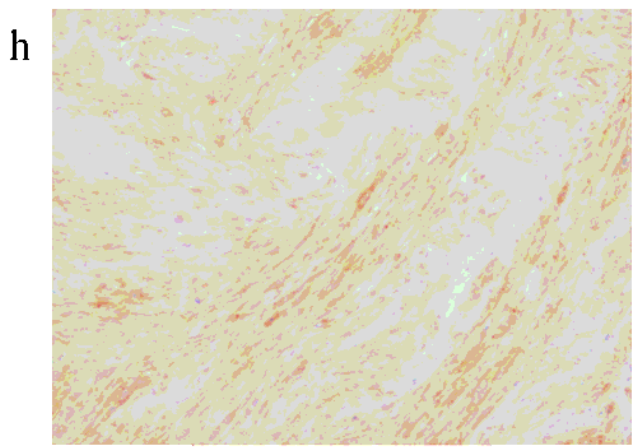

PD-L1: $\geq 50 \%$

\section{Figure 2}

Representative histological and immunohistochemical features: (a) pleomorphic and haphazard. (b) Bizarre tumor giant cell. (c-e) Intensity score (IS) 0 , IS 1 , and IS 2 is represented in order from left. f-h, PDL1 (<1\%), PD-L1 (1\% $\leq$ and $<50 \%)$, and PD-L1 ( $\geq 50 \%)$. 
a

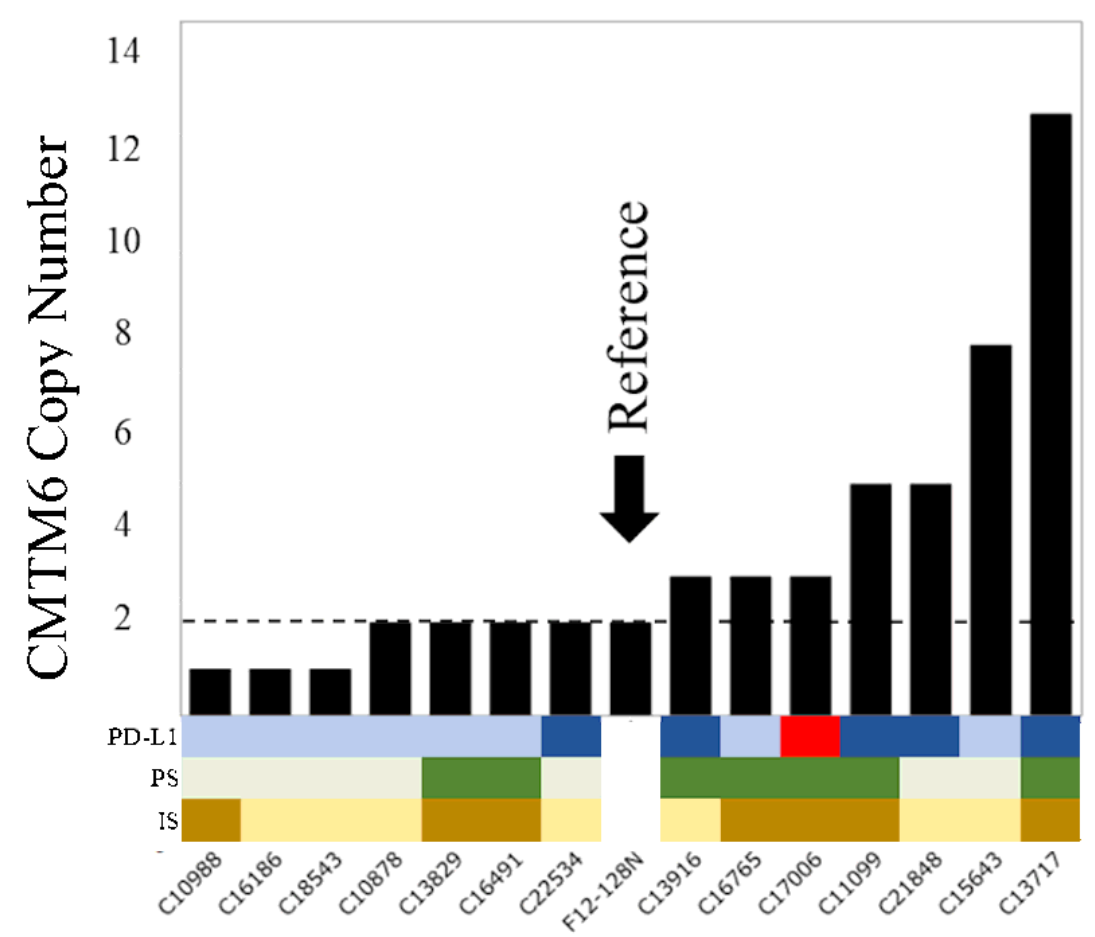

PD-L1

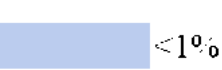

$1^{\circ} 0 \leq, 50^{\circ} \circ$

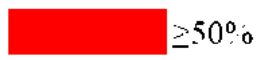

C.MTM6

$\mathrm{PS} \Omega 2$

$P S \geq 3$

IS $\_1$

IS $=3$

$\mathrm{b}$

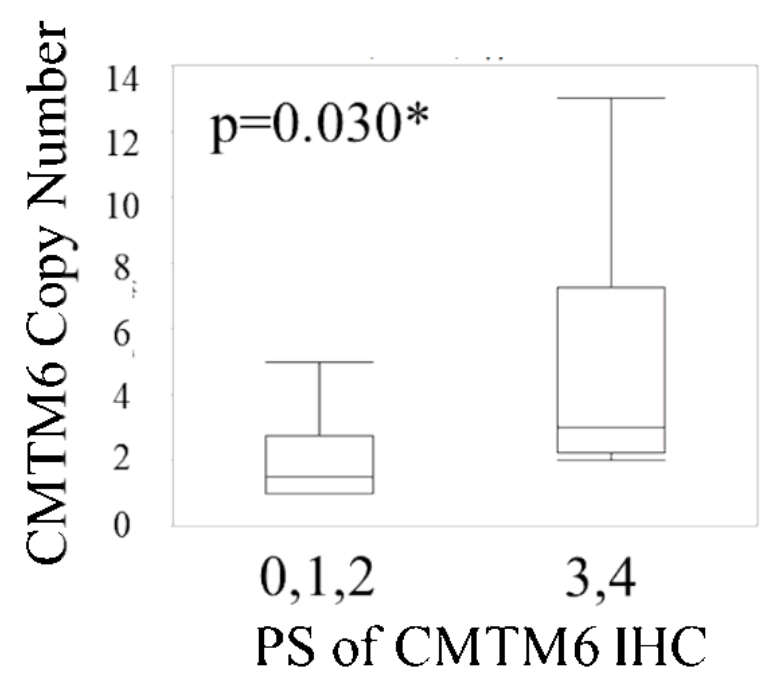

$\mathrm{c}$

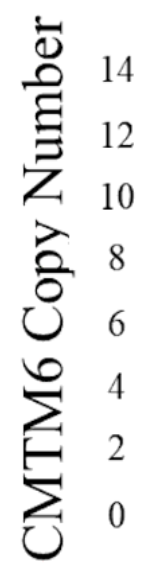

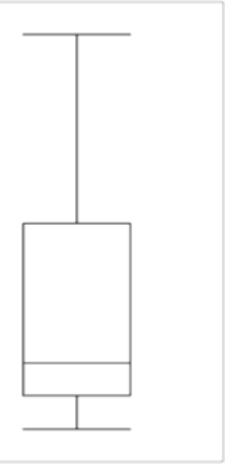

\section{$0,1 \quad 2$}

IS of CMTM6 IHC

Figure 3

(a) The result of copy number analysis. (b, c) The relationship between CKLF-like MARVEL transmembrane domain containing 6 (CMTM6) copy number and CMTM6 proportion score (PS) or intensity score (IS). 
d
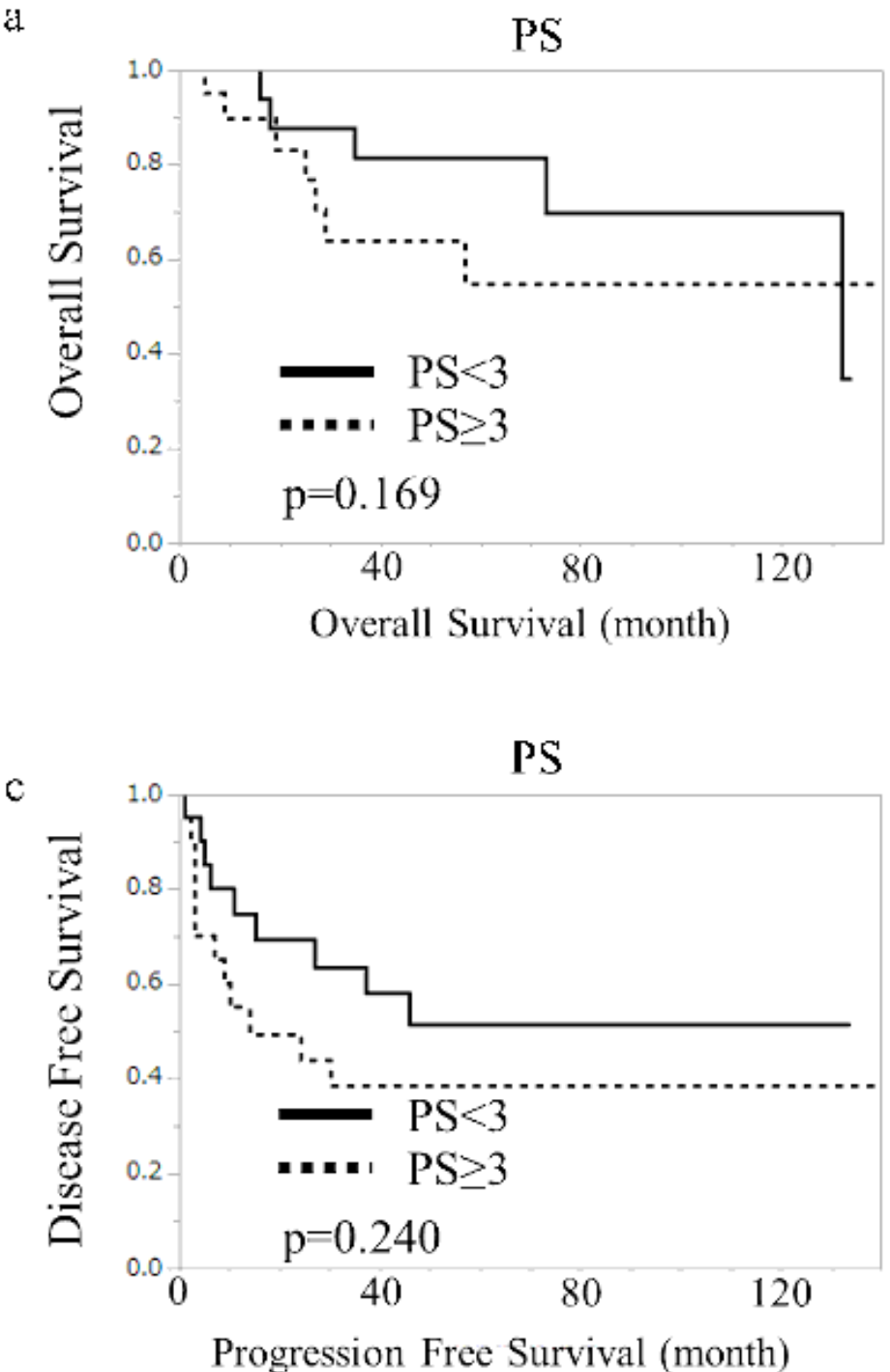

b

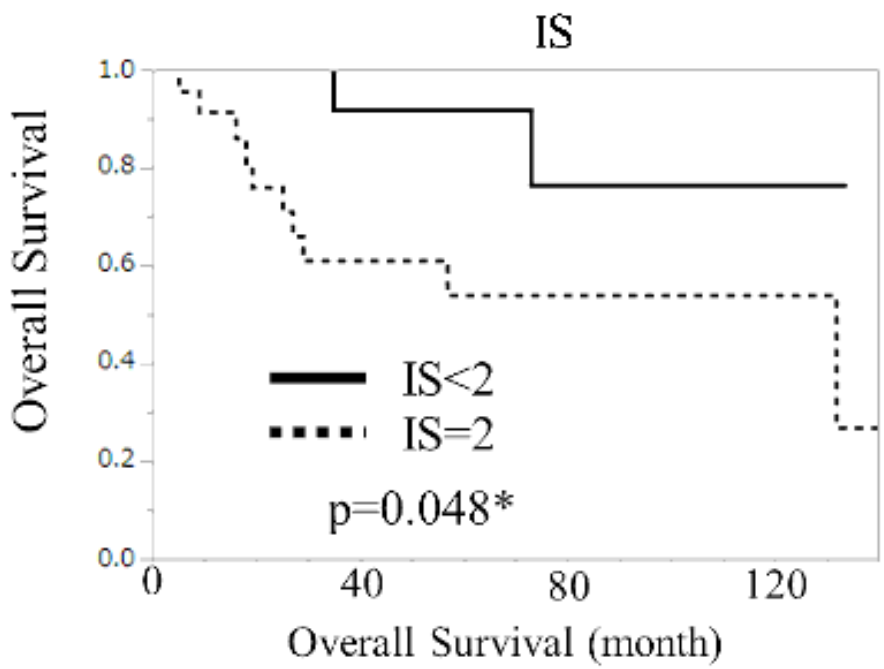

$\mathrm{d}$

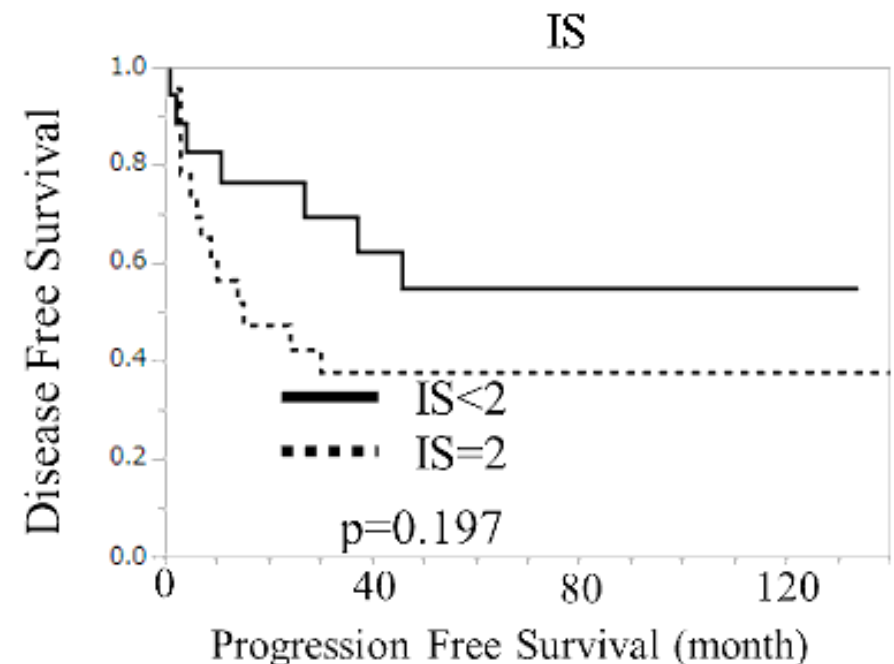

\section{Figure 4}

The outcome of survival analysis. Prognostic information for 41 cases was available. (a) Overall survival and proportion score (PS) of CKLF-like MARVEL transmembrane domain containing 6 (CMTM6). (b) Overall survival and intensity score (IS). (c) Disease-free survival and PS of CMTM6. (d) Disease-free survival and IS of CMTM6. 


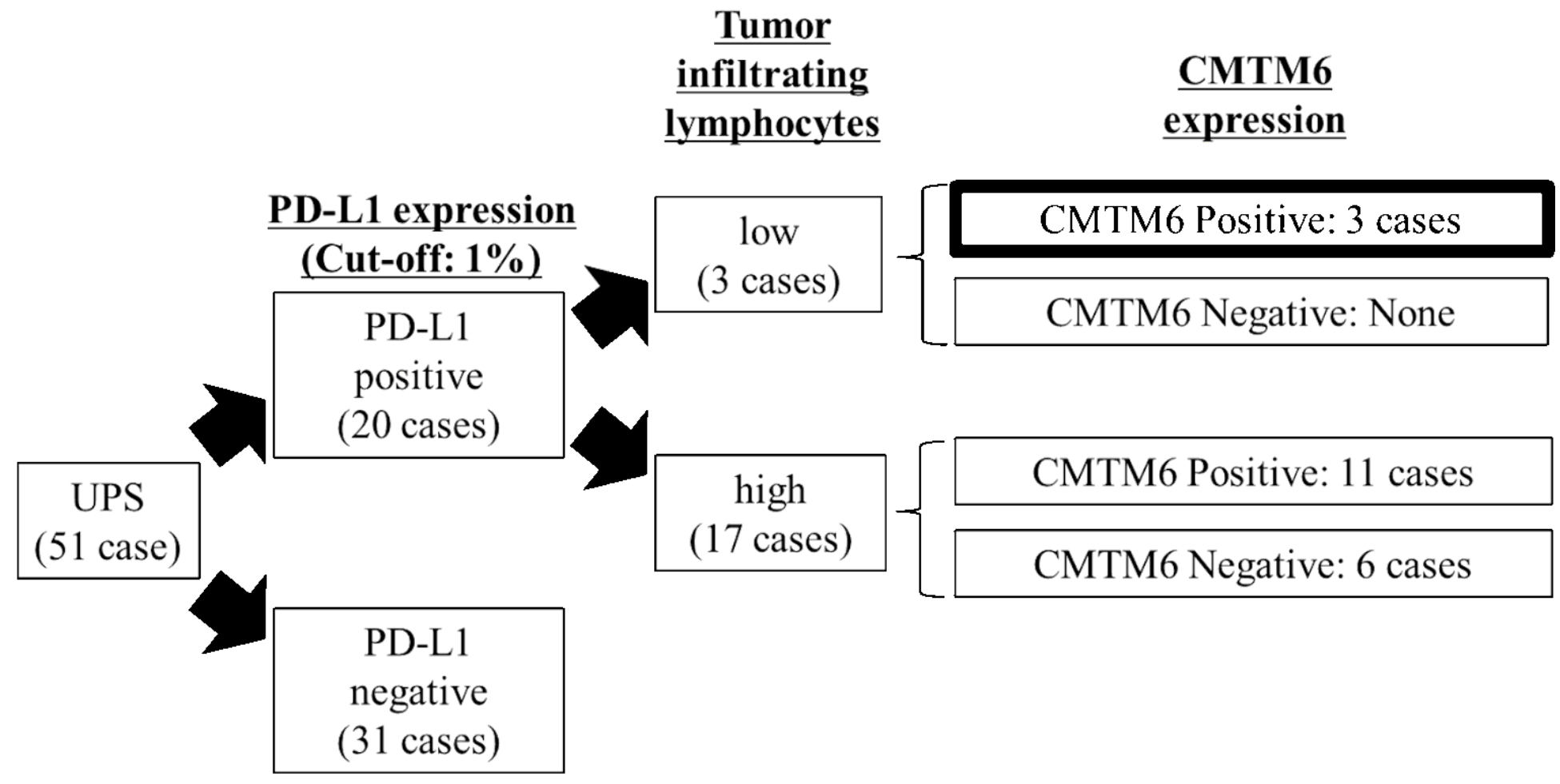

\section{Figure 5}

Chart subgrouping undifferentiated pleomorphic sarcoma (UPS) according to PD-L1 and CKLF-like MARVEL transmembrane domain containing 6 (CMTM6) expression and whether the number of tumorinfiltrating lymphocytes (TILs) was high. 